\title{
CORONAL MASS EJECTIONS AND SOLAR RADIO EMISSIONS
}

\author{
N. Gopalswamy*
}

\begin{abstract}
Three types of low-frequency nonthermal radio bursts are associated with coronal mass ejections (CMEs): Type III bursts due to accelerated electrons propagating along open magnetic field lines, type II bursts due to electrons accelerated in shocks, and type IV bursts due to electrons trapped in post-eruption arcades behind CMEs. This paper presents a summary of results obtained during solar cycle 23 primarily using the white-light coronagraphic observations from the Solar Heliospheric Observatory (SOHO) and the WAVES experiment on board Wind.
\end{abstract}

\section{Introduction}

Coronal mass ejections (CMEs) are associated with a whole host of radio bursts caused by nonthermal electrons accelerated during the eruption process. Radio bursts at low frequencies $(<15 \mathrm{MHz})$ are of particular interest because they are associated with energetic CMEs that travel far into the interplanetary (IP) medium and affect Earth's space environment if Earth-directed. Low frequency radio emission needs to be observed from space because of the ionospheric cutoff (see Fig. 1), although some radio instruments permit observations down to a few MHz [Erickson 1997; Melnik et al., 2008]. Three types of radio bursts are prominent at low frequencies: type III, type II, and type IV bursts, all due to nonthermal electrons accelerated during solar eruptions. The radio emission is thought to be produced by the plasma emission mechanism [Ginzburg and Zheleznyakov, 1958], involving the generation of Langmuir waves by nonthermal electrons accelerated during the eruption and the conversion of Langmuir waves to electromagnetic radiation. Langmuir waves scattered off of ions or low-frequency turbulence result in radiation at the fundamental (or first harmonic) of the local plasma frequency. Two Langmuir waves can also coalesce to produce electromagnetic waves at twice the local plasma frequency (second harmonic emission). The radio bursts thus provide important diagnostics of the solar eruption and the ambient medium through which the solar disturbances propagate.

The radio dynamic spectrum in Fig. 1 from the Radio and Plasma Wave (WAVES) experiment on board the Wind spacecraft [Bougeret et al., 1995] shows the three types

* NASA Goddard Space Flight Center, Greenbelt, MD 20771, USA 

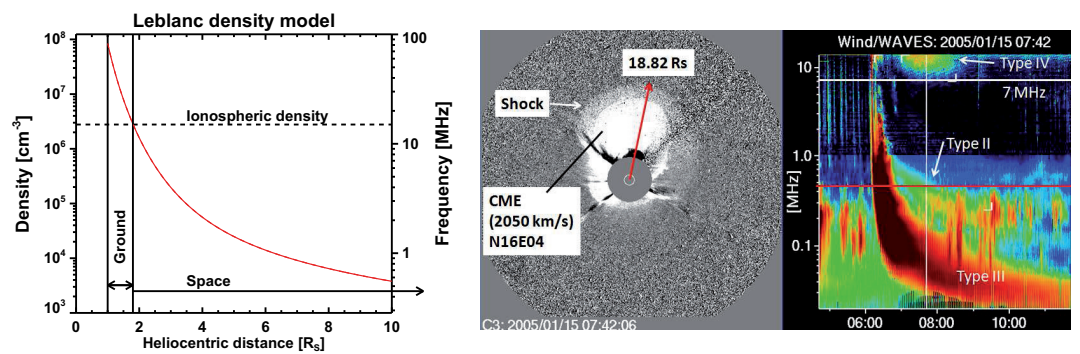

Figure 1: The Leblanc et al. [1998] density model (left). Radio phenomena occurring within (above) 2 Rs can be observed from ground(space) because of the ionospheric cutoff at $\sim 15$ MHz. A fast $(2050 \mathrm{~km} / \mathrm{s}) \mathrm{CME}$ observed by SOHO/LASCO on 2005 January 15 (middle) and the associated radio bursts of type II, type III, and type IV observed by Wind/WAVES (right).

of radio bursts, associated with an energetic eruption involving a major solar flare (soft X-ray flare size M8.6) and a fast CME observed by the Large Angle and Spectrometric Coronagraph (LASCO) [Brueckner et al., 1995] on board the Solar and Heliospheric Observatory (SOHO) mission. The type III burst is due to a relativistic electron beam propagating along open magnetic field lines. The intensity of the type III burst plotted in the frequency (f) - time (t) plane (known as the dynamic spectrum) shows a fast drift because of the high speed of the electron beam. The type II burst has a smaller drift rate (df/dt) because it originates from the front of the CME-driven shock. The type IV burst in Fig. 1 has a lowest frequency of $\sim 7 \mathrm{MHz}$, which means the loops that host the radio burst rise to a heliocentric distance of $\sim 2.4$ Rs, according to the Leblanc et al. (1998) density model shown in Fig. 1. Other density models give slightly different heights where the $7 \mathrm{MHz}$ plasma level occurs. For example, the Newkirk (1967) model gives a heliocentric distance between 2.5 and 3 Rs. Comparing CME images and the radio dynamic spectrum one can infer the rough heliocentric distance at which the type II radio bursts originate. The diffuse structure surrounding the CME flux rope in Fig.1 is thought to be the shock that produces the type II burst. The CME has a speed of $2050 \mathrm{~km} / \mathrm{s}$ and hence is expected to drive a shock of similar speed. When the shock is at a distance of $18.82 \mathrm{Rs}$ in the sky plane, the type II burst occurs at $450 \mathrm{kHz}$ (see Fig. 1). If the emission is at the fundamental of the plasma frequency,

$$
f[M H z]=f_{p}=9 \times 10^{-3} \sqrt{n(r)},
$$

one can get the electron density $n(r)$ as $\sim 2500 \mathrm{~cm}^{-3}$ at a heliocentric distance $\mathrm{r}$. The slope of the type II burst $(d f / d t)$ can be converted to the shock speed if we know the density scale height $H=\left[n^{-1} d n / d r\right]^{-1}$ in the ambient medium (see e.g., Mann and Klassen, 2005):

$$
(1 / f) \mathrm{d} f / \mathrm{d} t=V \cos \theta / 2 H,
$$

where $\theta$ is the angle between the radial direction and the direction of source motion. The left hand side of eq. (2) is sometimes referred to as the normalized drift rate, with $f$ taken as the central frequency of the range over which $d f / d t$ is measured. 

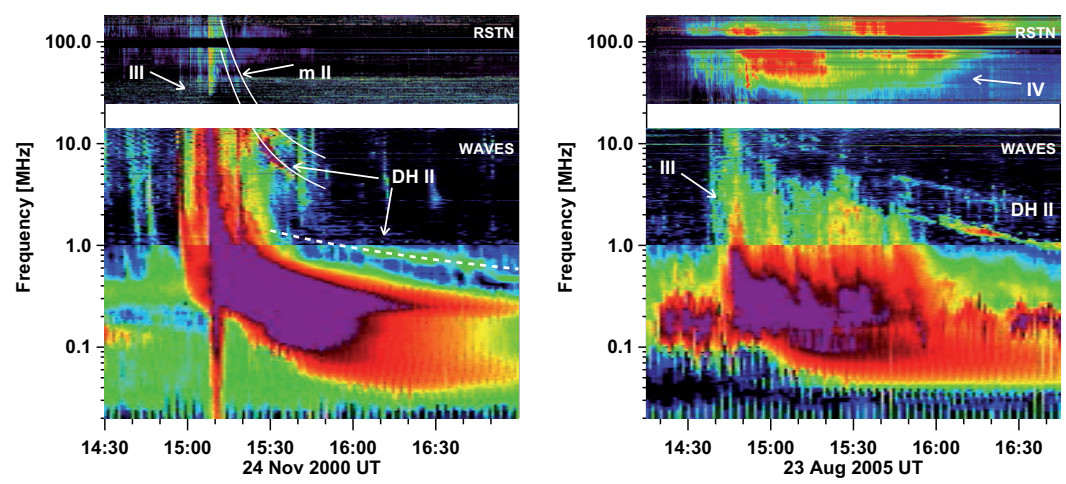

Figure 2: Composite dynamic spectrum from the ground based Radio Solar Telescope Network (RSTN) and Wind/WAVES observations showing Type III bursts on 2000 Nov 24 (left) and 2005 Aug 23 (right); the type III activity in both events is quite extended with durations of 30 and $70 \mathrm{~min}$, respectively. Both the bursts were associated with SEPs and major flares (X class for the November event and $M$ class for the August event). The type IV burst during the Aug 2005 event starts declining when the type III burst ends. Model fits to the type II burst components are also shown, which are discussed in section 3.

\section{Type III Bursts}

Type III bursts were first discovered in the frequency range 500 to $10 \mathrm{MHz}$ [Wild, 1950] and then at lower frequencies using space radio instruments [see the review by Gopalswamy, 2004a and references therein]. Type III bursts are thought to be produced by electron beams propagating along open magnetic field lines via the plasma emission mechanism. There are three low-frequency variants of type III bursts that originate in the interplanetary (IP) medium [Gopalswamy, 2004a]: (i) isolated type III bursts from flares and small-scale energy releases, (ii) complex type III bursts during CMEs, and (iii) type III storms. Here we are interested in the complex type III bursts, first identified in the ISEE-3 data by Cane et al. [1981], who named them as "shock accelerated (SA) events" because of the association with metric type II bursts. Kundu and Stone [1984] questioned the shockacceleration hypothesis and suggested that the electrons were accelerated in the flare site. Cane and Stone [1984] changed the terminology of SA events from "shock-accelerated" to "shock-associated" to allow for the possibility that the electrons responsible for these bursts may not physically originate from the associated shock [see Bougeret et al., 1998 for a recent discussion on the terminology]. The Wind/WAVES data in the decameter hectometric $(\mathrm{DH})$ wavelength range have filled the gap that existed between the ground $(>10 \mathrm{MHz})$ and space $(<2 \mathrm{MHz})$ based observations, so the connection of SA events to their coronal components has become much clearer. However, the controversy concerning the origin of the electrons responsible for the complex type III bursts still remains unresolved. Reiner et al. [2000] referred these bursts as complex type III bursts and strongly argued that the electrons are accelerated at the flare site. On the other hand, Dulk et al. [2000] studied eight type III bursts and concluded that the electrons are shock 

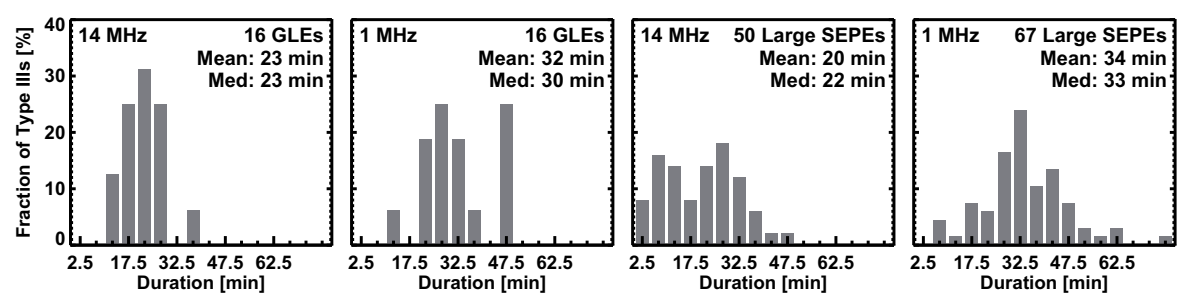

Figure 3: Durations of type III bursts associated with large SEP events (SEPEs) with GLE and non-GLE events shown separately.

accelerated. Gopalswamy et al. [2000] brought the CME-driven shocks into the picture: except for a slow filament eruption event and a backside event, all the CMEs they studied had long-duration type III bursts. In a recent study, Gopalswamy and Mäkelä [2010a] reported a complex type III burst that was associated with a fast and wide CME that lacked a type II burst. This event clearly questions the term "SA event" because there is neither shock acceleration nor shock-association. Therefore, the term "complex type III burst" is preferable.

Figure 2 shows two complex type III bursts associated with type II bursts at metric and longer wavelengths. The 2000 November 24 event has type III burst components starting at very high frequencies $(180 \mathrm{MHz})$ followed by lower frequency components that seem to originate from the type II burst. New bursts in the type III activity stop to appear beyond 15:30 UT when the DH type II burst is around $7 \mathrm{MHz}$, which drifts down to $\sim 4 \mathrm{MHz}$. The DH part has fundamental - harmonic structure. Another component of the type II burst starts around $1 \mathrm{MHz}$ at 15:55 UT. In the 2005 August 23 event, the type III burst seems to be originating entirely from the fundamental component of the type II burst between 14 and $2 \mathrm{MHz}$. Fast CMEs were associated with both the bursts $(1425 \mathrm{~km} / \mathrm{s}$ and $1929 \mathrm{~km} / \mathrm{s}$ for the November 2000 and August 2005 CMEs, respectively). When new bursts stopped appearing in the type III activity, the CMEs were still close to the Sun and driving shocks. Dulk et al. [2000] also noted that type III bursts typically ended when the associated type II bursts reached 5-6 MHz. It is not clear why the type III bursts ended so soon if the shocks were accelerating the type III electrons. One possibility is the suggestion by Bougeret et al. [1998] that the type III bursts are turned on when the electrons accelerated in the flare reconnection region reach the CME-driven shock. In the August 2005 event, the appearance of new components in the type III activity stops when the lowest frequency of the type IV emission suddenly starts increasing, suggesting a closer connection to the flare reconnection site.

\subsection{Complex Type III Bursts and SEP Events}

Kahler et al. [1986] reported that large solar energetic particle (SEP) events were well correlated with the complex type III events irrespective of the eruption longitude. Recently, Cane et al. [2002] also concluded that $\sim 20 \mathrm{MeV}$ proton events that lasted longer than $36 \mathrm{hr}$ were linked to the complex type III bursts with an average $14 \mathrm{MHz}$ duration of 

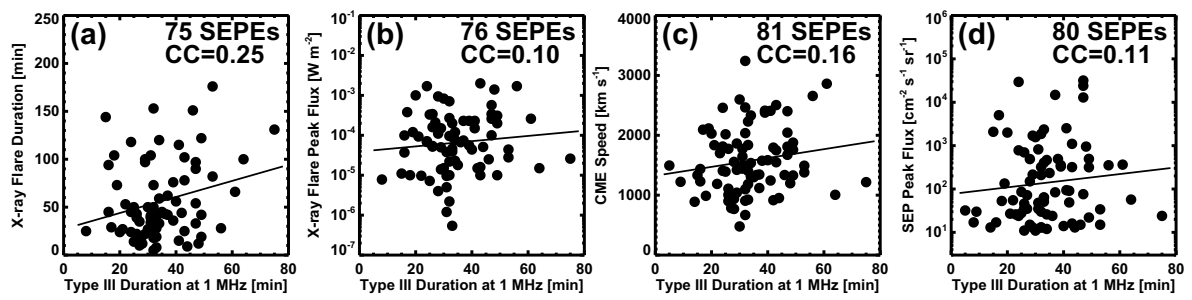

Figure 4: The relation between the type III burst duration at $1 \mathrm{MHz}$ and (a) soft X-ray flare duration, (b) soft X-ray flare size, (c) CME speed, and (d) SEP peak intensity.

20 min. Gopalswamy and Mäkelä [2010a] reported the distribution of complex type III burst durations at 14 and $1 \mathrm{MHz}$ (see Fig. 3) associated with large SEP events (particle intensity exceeding 10 particle flux units (pfu) in the $>10 \mathrm{MeV}$ GOES energy channel; $1 \mathrm{pfu}=1 \mathrm{particle} / \mathrm{cm}^{2} / \mathrm{s} / \mathrm{sr}$ ). The SEP events with ground level enhancements (GLEs) show a symmetric distribution with average durations of 23 and $32 \mathrm{~min}$, respectively at 14 and $1 \mathrm{MHz}$. For the non-GLE SEP events, the average durations are not very different: $20 \min (14 \mathrm{MHz})$ and $34 \mathrm{~min}(1 \mathrm{MHz})$. Gopalswamy and Mäkelä [2010a] also reported a long-duration (25 min at $14 \mathrm{MHz}$ and $28 \mathrm{~min}$ at $1 \mathrm{MHz}$ ) complex type III burst associated with a fast $(977 \mathrm{~km} / \mathrm{s})$ partial halo CME, but was not associated with an SEP event. This is an important counter example, which suggests that the statistical association between complex type III bursts and SEP events does not indicate a physical relationship. The complex type III burst also lacked a type II burst and an IP shock, consistent with the lack of SEP event under the shock paradigm. Since there is no shock, the complex type III burst cannot be called an SA event (neither shock-accelerated nor shock-associated). Cliver and Ling [2009] found that neither the intensity nor the duration of the associated complex type III bursts could distinguish between the gradual and impulsive SEP events. They concluded that the complex type III bursts can be thought of as a consequence of a fast CME, which when driving a strong shock produces type II and SEP events. Major flares without CMEs are generally not associated with type III bursts at long wavelengths [Gopalswamy et al., 2009a; Klein et al. 2010]. The absence of long wavelength radio emission in such events was used by Klein et al. (2010) as a signal that no SEP event is to be expected in the interplanetary medium. However, as Gopalswamy and Mäkelä[2010a] showed, the presence of a complex type III burst does not necessarily indicate a SEP event if there is no accompanying type II burst.

If the electrons responsible for the type III bursts originate from the flare reconnection, they may indicate some relationship with the soft X-ray flare. If the type III electrons are from the CME-driven shock, one would expect some relationship between the type III duration and CME speed. Finally, if the SEPs and type III electrons have common acceleration process, there should be some correlation between the type III duration and SEP intensity [see e.g., MacDowall et al., 2003]. In order to see these, we have made several scatter plots in Fig. 4. All the correlations are rather poor. The highest correlation is between the soft X-ray flare duration and the type III burst duration (0.25). Neither the flare nor the shock process proxies have a good correlation with the type III duration. 
Considering the fact that the type III burst intensity is similar for the large impulsive and gradual SEP events [Cliver and Ling, 2009], it is not clear if the type III bursts could provide additional clue to the particle acceleration process during solar eruptions.

\section{Type II Bursts}

Type II bursts were first identified by Payne-Scott et al. [1947], who recognized the importance of mass motion for these bursts. Wild and McCready [1950] classified them as type II bursts to distinguish from the fast-drifting type III bursts. In the IP medium, type II observations were first made by the IMP-6 [Malitson et al., 1973] and Voyager [Boischot et al., 1980] missions. The ISEE-3 spacecraft observed many IP type II bursts in the frequency range $30 \mathrm{kHz}$ to $2 \mathrm{MHz}$ [Cane et al., 1987; Lengyel-Frey and Stone, 1989]. Finally, the Wind/WAVES experiment filled the gap between $2 \mathrm{MHz}$ and the $14 \mathrm{MHz}$, thus enabling a complete view of type II bursts [Gopalswamy et al., 2005].

Uchida [1960] suggested that the type II bursts are produced by MHD shocks in the corona. The origin of shocks responsible for type II bursts has been controversial: flare blast waves or CME-driven shocks [see Gopalswamy, 2006 for a detailed discussion on this issue]. The blast wave scenario can be traced to the observation that a third of metric type II bursts were not associated with CMEs [Sheeley et al., 1984]. However, a detailed examination revealed that most of those CMEless type II bursts originated from close to the disk center [Cliver et al., 1999]. Coronagraphs, by their very nature are ill-positioned to observe CMEs from the disk center, but the CMEs can be inferred from the large-scale EUV disturbances [Gopalswamy et al., 2001a]. In fact, when type II bursts originating from close to the limb are considered, almost all of them were associated with CMEs [Munro et al., 1979; Gopalswamy et al., 2005]. The occasional reports invoking blast waves for type II bursts have also recognized the presence of slower CMEs $(<500 \mathrm{~km} / \mathrm{s})$ [Magdalenić et al., 2010]. Finally, even X-class flares are not associated with type II bursts if they lack CMEs, further pointing to the importance of CMEs for the occurrence of type II bursts [Gopalswamy et al., 2009a]. Klein et al. [2010] also considered X-class flares without CMEs, but included two events rejected by Gopalswamy et al. [2009a] and claimed coronal shocks without CMEs. Both these events were disk events and had new material appearing in the corona around the time of the type II bursts. Therefore, these two flares (and type II bursts) cannot be considered as CMEless. The shock origin controversy concerns only the metric type II bursts because all type II bursts below 2 $\mathrm{MHz}$ [Cane et al., 1987] or in the 1-14 MHz range [Gopalswamy et al., 2001b] have been associated with CMEs irrespective of their source position on the Sun.

Figure 5 shows the Wind/WAVES dynamic spectrum consisting of several components of a type II burst and the associated fast CME $(1813 \mathrm{~km} / \mathrm{s})$. The metric $(\mathrm{m})$ type II burst starts near $180 \mathrm{MHz}$ and ends near $7 \mathrm{MHz}$ in the decameter - hectometric (DH) domain. Another slow-drifting feature starts around $6 \mathrm{MHz}$ and continues into the kilometric $(\mathrm{km})$ domain. The overall pattern of the type II components is similar to the one during the 2000 November 24 event shown in Fig. 2. Events like these have been interpreted in two ways: 1 . The $\mathrm{DH}$ - km component is caused by the CME-driven shock, while the source of the $\mathrm{m}$ - DH component is unknown or the flare blast wave [see, e.g., Cane and Erickson, 


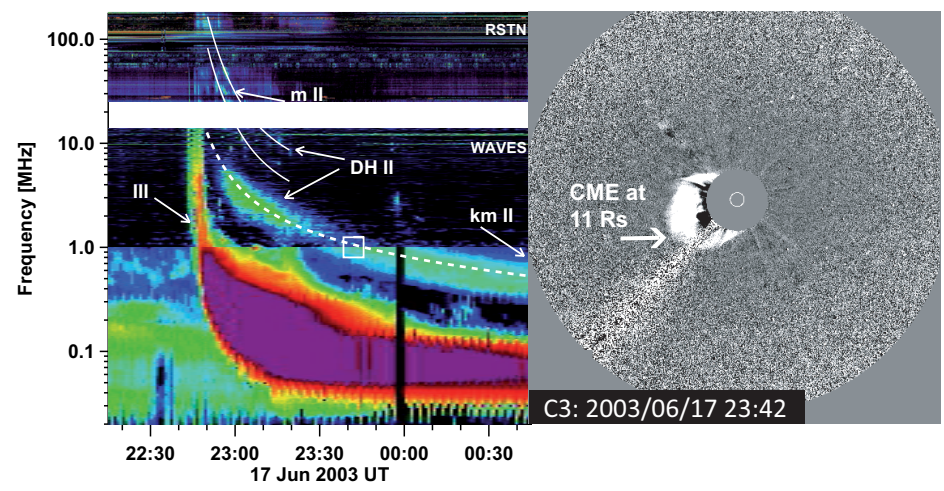

Figure 5: (left) Type II burst components in metric (m), decameter - hectometric (DH) and kilometric $(\mathrm{km})$ wavelength domains. (right) the CME driver had its leading edge at $11 \mathrm{Rs}$ at 23:42 UT when the type II burst reached a frequency of $1 \mathrm{MHz}$ (indicated by the white square).

2005]. 2. Both the components are caused by the same shock, the $\mathrm{m}$ - DH coming from the shock flanks, while the DH - km component coming from the shock nose [Raymond et al., 2000]. The $\mathrm{m}-\mathrm{DH}$ and DH - km components in the 2003 June 17 event (Fig. 5) start nearly simultaneously (around 22:54 UT), with no harmonic relationship between the two. This is possible if the shock is curved with the flanks in the high density region (low in the corona) and the nose in the low density region (higher up in the corona). Let us see if the type II components fit this scenario. When the CME first appeared, the leading edge was already beyond the $\mathrm{LASCO} / \mathrm{C} 2$ field of view (FOV), because of the high speed $(1813 \mathrm{~km} / \mathrm{s})$. The shock structure surrounding the CME had a nose speed of $1869 \mathrm{~km} / \mathrm{s}$. From the fundamental component of the m-DH type II, one can get the normalized drift rate between 25.5 MHz (22:56:00 UT) and 10.8 MHz (23:02:30 UT) as $2.08 \times 10^{-3} \mathrm{~s}^{-1}$ for the mid frequency of $18.15 \mathrm{MHz}$. Assuming that the radio source is located at $\theta=45^{\circ}$ from the nose, we get the local shock speed as $1322 \mathrm{~km} / \mathrm{s}$. Substituting these two quantities into eq. (2), we get the scale height $H=3.2 \times 10^{5} \mathrm{~km}$ or $0.46 \mathrm{Rs}$. The Saito et al. (1977) model with a multiplier of 3.7 gives this scale height if the source is located at a heliocentric distance of $2.42 \mathrm{Rs}$ at 22:59 UT. These parameters clearly fit the fundamental - harmonic structure of the m-DH type II burst shown in Fig. 5. Assuming that the DH - km type II burst comes from the CME nose, we can use the observational constraint that the shock stands at a heliocentric distance of 7.06 Rs at 23:18 UT from LASCO/C3 image. At this time, the radio dynamic spectrum shows that the type II burst is at $1.8 \mathrm{MHz}$, which is the local plasma frequency at a heliocentric distance of 7.06 Rs, if we assume fundamental emission. The Saito et al. (1977) density model with a multiplier of 4.5 matches this plasma level and readily fits the DH - km type II burst as shown in Fig. 5. The fits shown in Fig. 2 for the 2000 November 24 event follow a similar 

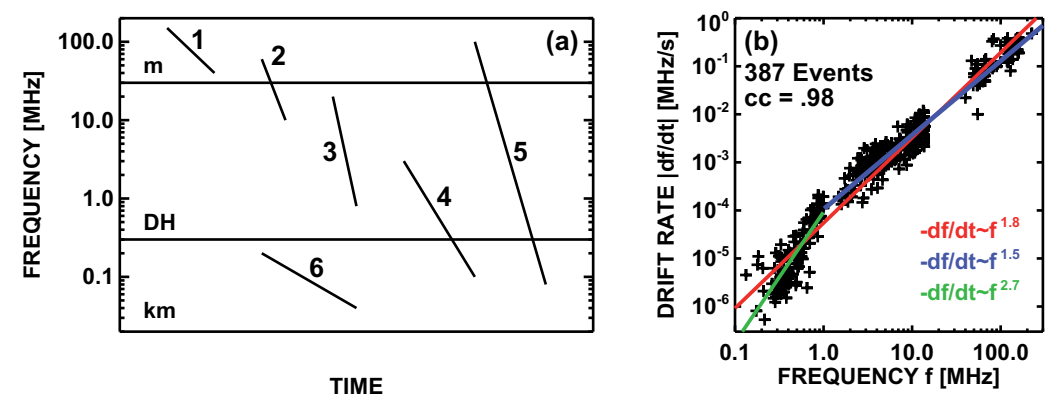

Figure 6: (a) A schematic dynamic spectrum illustrating the variants of type II bursts: 1. Purely $\mathrm{m}, 2 . \mathrm{m}-\mathrm{DH}$, 3. $\mathrm{DH}, 4$. $\mathrm{DH}-\mathrm{km}, 5 . \mathrm{m}-\mathrm{km}$, and 6 . Purely $\mathrm{km}$. (b) the drift rate $(d f / d t)$ dependence on the emission frequency $(f)$ in the $\mathrm{m}, \mathrm{DH}$, and $\mathrm{km}$ domains using data from various sources: $m$ from Potsdam data, $D H$ and $\mathrm{km}$ from Wind/WAVES RAD1 and RAD2 data as well as data from ISEE-3 (387 events in all). The scatter plot has a correlation coefficient of $\sim 0.98$ for the whole frequency range. $\mathrm{m}-\mathrm{DH}$ and $\mathrm{DH}-\mathrm{km}$ data points taken by themselves show deviations from the regression line (see text).

analysis.

The second interpretation is thus highly likely because at a given time the CME-driven shock spans a large range of densities. The slope of the $\mathrm{m}-\mathrm{DH}$ and $\mathrm{DH}-\mathrm{km}$ components are also different because the nose and flanks have different speeds and move at different angles to the radial direction. Furthermore, the CME speed and the Alfven speed also change with the radial distance, so the same shock has different strength and Mach number at different locations [Gopalswamy et al., 2001a; Mann et al., 2003].

Type II bursts have a high-frequency cutoff at $\sim 150 \mathrm{MHz}$, attributed to the fact that the Alfven speed is very high in the core of the active regions and falls off rapidly as one goes away from the active region radially as well as laterally [Gopalswamy et al., 2001a]. Considering only type II bursts in which the fundamental component can be clearly identified, Gopalswamy et al. [2005] obtained a distribution of metric type II starting frequencies that had an average starting frequency of $\sim 101 \mathrm{MHz}$. The last bin in the distribution was $250 \mathrm{MHz}$. Only $<8 \%$ of the events had type II starting frequencies above $150 \mathrm{MHz}$. Occasionally one observes type II bursts at higher frequencies [see, e.g. Pohjolainen et al. 2008], but these are exceptions. Some type II bursts start and end within the metric $(\mathrm{m})$ domain $(>20 \mathrm{MHz})$ with no counterpart at lower frequencies. These are known as purely $\mathrm{m}$ type II bursts. Others start and end within DH wavelength domain (14-1 MHz). Bursts starting and ending in the $\mathrm{km}$ wavelength domain are referred to as purely $\mathrm{km}$ type II bursts. Some bursts have counterparts in all the wavelength domains ( $\mathrm{m}$ - $\mathrm{km}$ type II bursts). In many events, one finds an $\mathrm{m}$ or $\mathrm{m}$ - DH component and a DH - $\mathrm{km}$ component as separate features, but starting nearly simultaneously (as in Figures 2 and 5). Figure 6 (a) summarizes these variants of type II bursts.

The close connection between CMEs and type II bursts can also be seen from the hierarchi- 
cal relation between CME energy and the wavelength range of type II bursts [Gopalswamy et al., 2005]. The average speed of CMEs progressively increases as one goes from purely $\mathrm{m}$ to DH to $\mathrm{m}$ - $\mathrm{km}$ type II bursts: 610,1068 , and $1490 \mathrm{~km} / \mathrm{s}$, respectively. The halo fraction (average width of non-halo CMEs) also increases from $3.8 \%\left(85^{\circ}\right.$ ) for $\mathrm{m}$ type II bursts, $48 \%\left(130^{\circ}\right)$ for DH type II bursts and $71 \%\left(171^{\circ}\right)$ for $\mathrm{m}-\mathrm{km}$ type II bursts. The average speed, width, and halo CME fraction are $480 \mathrm{~km} / \mathrm{s}, 45^{\circ}$, and $3 \%$, respectively for the general population of CMEs [see, e.g., Gopalswamy, 2004b]. The purely km type II bursts have speeds (average $\sim 539 \mathrm{~km} / \mathrm{s}$ ) similar to those of the purely $\mathrm{m}$ type II bursts, but their acceleration is negative [Gopalswamy, 2006]. This means that the CMEs associated with the purely $\mathrm{km}$ type II bursts continue to accelerate and drive shocks only beyond $\sim 10$ Rs. If the metric type II bursts are not related to the associated CMEs, there is no reason as to why they should have the hierarchical relationship.

Another property of the type II bursts is the frequency $(f)$ dependence of the drift rate $(\mathrm{d} f / \mathrm{d} t)$ measured over different wavelength domains [Vršnak et al., 2001; Aguilar Rodriguez et al., 2005; Gopalswamy et al., 2009b]:

$$
\mathrm{d} f / \mathrm{d} t \propto f^{\varepsilon},
$$

where $\varepsilon \sim 2$. Figure $6 \mathrm{~b}$ shows that $\varepsilon=1.8$ for frequencies from 0.1 to $250 \mathrm{MHz}$ (valid over six orders of magnitude in $d f / d t$ ). The $f-d f / d t$ relationship is referred to as the universal drift-rate spectrum [Gopalswamy et al., 2009b], which suggests that there must be something common for the type II bursts at different wavelength regimes: the CMEdriven shock. The exponent $\varepsilon$ can be readily derived assuming that the emission occurs at the local plasma frequency $\left(f_{p}\right)$ [Vršnak et al., 2001]. Equation (2) can be rewritten as,

$$
\mathrm{d} f / \mathrm{d} t=(V f / 2 n) \mathrm{d} n / \mathrm{d} r,
$$

with $V$ being the shock speed. If the radio source moves at an angle $\theta$ to the density gradient, then $V$ must be replaced by $V \cos \theta$. Assuming that the density falls off with distance according to: $n(r) \propto r^{-\alpha}$ and hence $f \propto r^{-\alpha / 2}$ so one can write equation (3) as

$$
|\mathrm{d} f / \mathrm{d} t| \propto V r^{-1-\alpha / 2} \propto V f^{1+2 / \alpha}
$$

In the IP medium, $\alpha \sim 2$, so the right hand side of Eq. (5) becomes $V f^{2}$ explaining the overall drift rate spectrum, provided $V$ is constant. Fig. $6 \mathrm{~b}$ shows that in the individual spectral domains, $\varepsilon$ deviates significantly from 2 : the $\mathrm{km}$ part shows a steeper slope $(\varepsilon \sim 2.7)$ while the $\mathrm{m}-\mathrm{DH}$ part has a shallower slope $(\varepsilon \sim 1.5)$ [see also Aguilar-Rodriguez et al. 2005]. These deviations can be explained if we note that $V$ is not constant: the speed rapidly increases near the Sun and then decreases for most of the CMEs that drive shocks. The speed variation can be introduced in Eq. (5) by assuming a radial dependence for $V$ :

$$
V \propto r^{-\beta} \text { or } V \propto f^{2 \beta / \alpha},
$$

resulting in

$$
\varepsilon=(\alpha+2 \beta+2) / \alpha .
$$

In the IP medium, $\alpha \sim 2$, so Eq. (6) becomes,

$$
\varepsilon=2+\beta .
$$



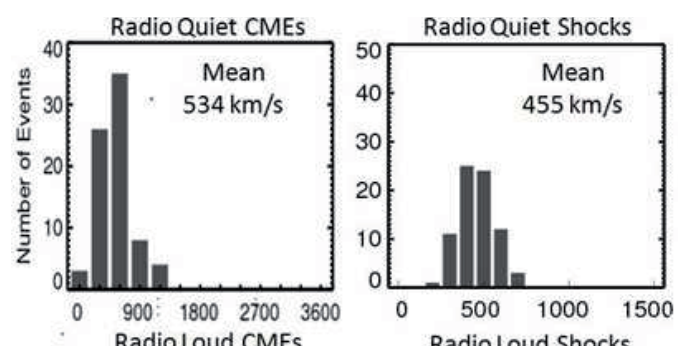

Radio Quiet CMEs
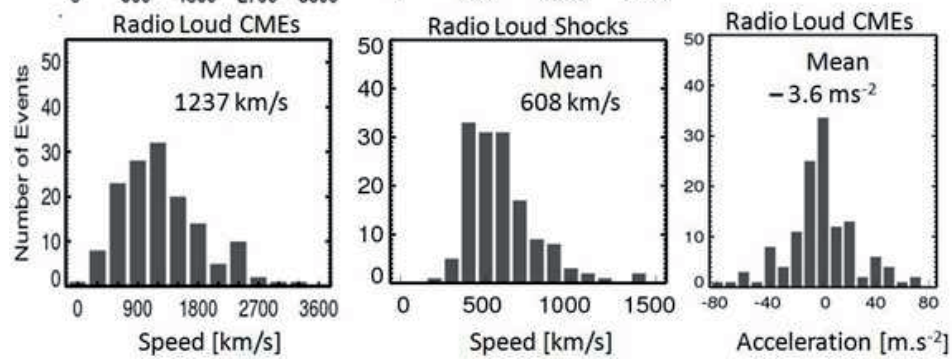

Figure 7: CME speeds (left), IP shock speeds (middle), and CME accelerations (right) for the radio-quiet and radio-loud events.

With $\varepsilon=2.7$ in the $\mathrm{km}$ domain (see Fig. $6 \mathrm{~b}$ ), we get $\beta=0.7$, which means that the CME speeds decline in the IP medium.

Things are more complicated in the metric and DH domains because both $\alpha$ and $\beta$ change with heliocentric distance. For a set of type II bursts in the metric domain, Gopalswamy et al. [2009b] found that $\varepsilon=1.07$. Since $\alpha \sim 6$ in the inner corona, one gets $\beta=-0.79$ (increasing speed). Individual CME events with observations in the metric domain did show such $\beta$ values [Gopalswamy et al., 2009b]. In the outer corona, $\alpha \sim 4$, so $\varepsilon=1.5$ (see Fig. 6) implies $\beta \sim 0$ because CMEs attain their maximum speed here and their speeds remain roughly constant before decelerating due to the drag force. The universal drift rate spectrum thus reflects the CME evolution consistent with the suggestion that all the type II bursts can be attributed to CME-driven shocks.

\subsection{Type II Bursts and IP Shocks}

Type II bursts are the earliest indicators of CME-driven shocks. Many of these shocks originating on the front-side of the Sun continue to survive in the IP space and are detected by spacecraft in the solar wind. Starting from IP shocks Gopalswamy et al. (2010b) found a surprisingly large fraction (34\%) of them to be radio-quiet (lacked type II emission). Radio-quiet and radio-loud shocks differed in a number of ways. 1. Radio-quiet shocks had an average CME speed of $\sim 535 \mathrm{~km} / \mathrm{s}$, compared to $\sim 1237 \mathrm{~km} / \mathrm{s}$ for the radio-loud ones; the in-situ shock speeds, on the other hand, were similar (see Fig. 7). The difference between the radio-quiet and radio-loud events seems to be erased as the CMEs exchange momentum with the ambient solar wind. 2. The radio-quiet CMEs accelerate on the 

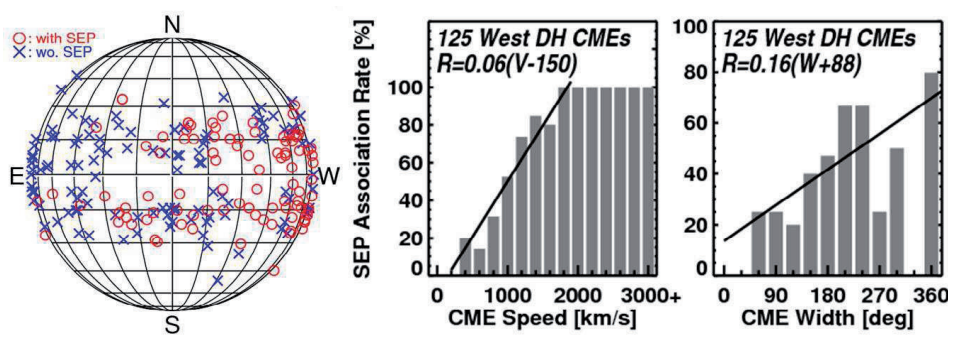

Figure 8: (left) CME sources of DH type II bursts (circles and crosses denoting events with and without $\geq 1$ pfu SEP events). (right) SEP association rate as a function of CME speed and width for western hemispheric CMEs with DH type II bursts.

average, while the radio-loud CMEs decelerate. The predominant acceleration found in the radio-quiet CMEs is somewhat similar to that in the CMEs associated with purely km type II bursts; the radio-quiet shocks are even weaker than the shocks responsible for the purely $\mathrm{km}$ type II bursts. 3. Among the radio-loud CMEs, some are radio loud only near the Sun (associated with purely metric type II bursts), some only near Earth (associated with purely kilometric type II bursts), and others throughout the IP medium. Shocks producing purely metric and purely kilometric type II bursts have the lowest CME kinetic energy, but have opposite sign of acceleration on the average. 4. Soft X-ray flares associated with radio-quiet shocks are generally weaker than those associated with radioloud shocks, consistent with the corresponding CME kinetic energies. 5. The CME source locations of radio-quiet shocks are concentrated near the central meridian, while those of radio-loud shocks are more widely distributed. This suggests that the radio-loud shocks are more extended than the radio-quiet ones.

\subsection{Type II Bursts and SEP Events}

Type II bursts are closely related to the SEP events because the same shock is responsible for both the phenomena. Studying a set of nearly 500 fast (speed $\geq 900 \mathrm{~km} / \mathrm{s}$ ) and wide (width $\geq 60^{\circ}$ ) CMEs Gopalswamy et al. [2008a,b] found that if a CME is radio quiet, it also lacked a large SEP event, suggesting the importance of a strong shock in producing an SEP event. Statistical studies have shown that nearly all large SEP events are accompanied by type II bursts [Gopalswamy 2003; Cliver et al., 2004]. CMEs associated with purely metric type II bursts are the weakest among radio-loud CMEs, so only $13 \%$ of them are associated with SEP events with intensity $\geq 1 \mathrm{pfu}$. If we consider CMEs with DH type II bursts (no metric type II burst), the association rate jumps to $42 \%$. Finally, when we consider CMEs with type II bursts in the metric and longer wavelength domains, the SEP association attains the maximum value of 56\% [Gopalswamy et al., 2008b]. The CME properties are nearly identical for $\mathrm{m}$ - $\mathrm{km}$ type II bursts and for large SEP events because the same shock produces the SEP event and the type II burst. Figure 8 shows the source locations of radio-loud CMEs with and without an associated SEP event. There is a clear western bias for CMEs associated with SEP events because 

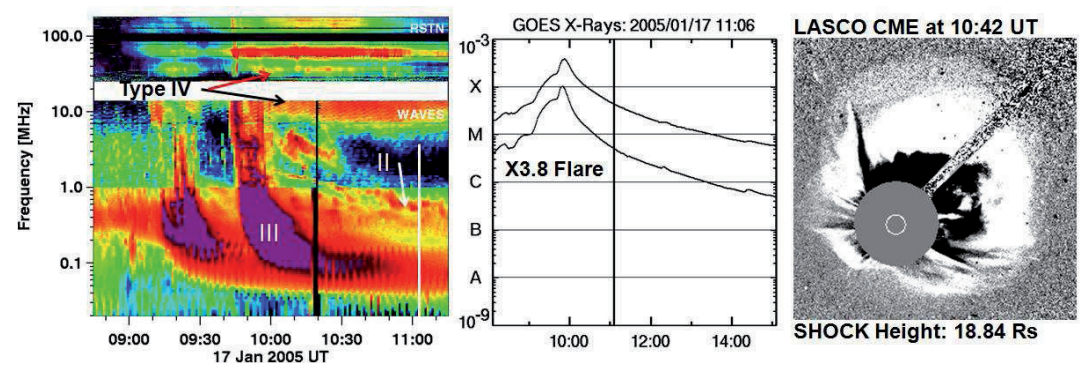

Figure 9: (left) Composite dynamic spectrum from RSTN and Wind/WAVES showing the type IV burst on 2005 January 17 extending from above $180 \mathrm{MHz}$ down to $\sim 3 \mathrm{MHz}$. Accompanying type II and type III bursts are marked. The vertical white line marks the time when the type IV is emitted at the lowest frequency. (middle) GOES soft X-ray light curve in two energy channels showing the X3.8 flare associated with the type IV burst. The vertical solid line marks the time when the type IV burst descends to the lowest frequency. (right) White-light CME near the time when the type $I V$ descended to the lowest frequency.

western sources are well connected to an Earth observer. Most radio-loud CMEs with their solar sources in the eastern hemisphere are not associated with SEP events. There are occasional eastern sources with SEP events and several western hemispheric sources without SEP events. These exceptions can be explained by the additional speed and width dependence of the radio-loud CMEs. The histograms in Figure 8 show that the SEP association rate reaches $100 \%$ only for the fastest CMEs (speeds $\geq 1800 \mathrm{~km} / \mathrm{s}$ ). Similarly, the association rate steadily increases with CME width, reaching a maximum of $80 \%$ for full halo CMEs.

\section{Type IV Bursts}

The low-frequency type IV bursts have not been studied extensively because the frequency window in which these bursts occur became available only after the launch of the Wind mission in 1994. Leblanc et al. [2000] reported on the 1998 May 2 type IV burst, which descended down to $\sim 7.5 \mathrm{MHz}$, similar to the one shown in Fig. 1. Gopalswamy [2004c] reported on a set of $12 \mathrm{DH}$ type IV bursts observed by Wind/WAVES during 1997 to 2002. They found that the DH type IV bursts are extensions of the well-known metric continua and lasted typically for $\sim 2$ hours at $14 \mathrm{MHz}$ with an average lowest frequency of $\sim 7.7 \mathrm{MHz}$. The type IV bursts are associated with very energetic CMEs (average speed $\sim 1200 \mathrm{~km} / \mathrm{s}$ ), confirming the earlier finding by Robinson [1986] for the continuum events at metric wavelengths. Chernov et al. [2007] studied fine structures in the low frequency type IV bursts observed by Wind/WAVES. Melnik et al. [2008] reported on the properties of type IV bursts observed by ground based radio telescopes operating in Ukraine in the frequency range $10-40 \mathrm{MHz}$.

Figure 9 shows a type IV burst extending from above $180 \mathrm{MHz}$ down to $\sim 3 \mathrm{MHz}$. The radio emission should originate from a heliocentric distance 3.5 to $4.5 \mathrm{Rs}$, depending 

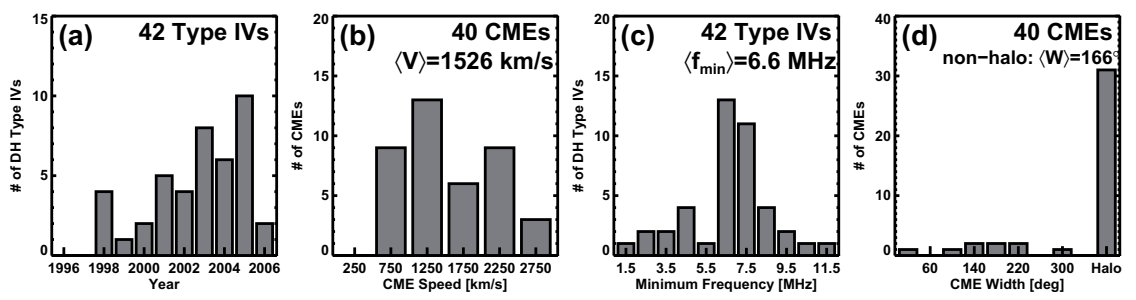

Figure 10: Statistics of type IV bursts: (a) annual rate, (b) speed distribution of associated CMEs, (c) distribution of minimum frequency, and (d) width distribution of the associated CMEs.

on whether the radio emission occurs at the fundamental or harmonic of the plasma frequency. When the type IV burst attains the lowest frequency, the IP type II burst occurs at frequencies well below $1 \mathrm{MHz}$, which means the shock is much farther away as can be inferred from the LASCO image in Fig. 9: the CME-driven shock is at a distance of 18.84 Rs in sky-plane projection (expected to be larger when projection corrections are made). Thus there is a separation of at least $14 \mathrm{Rs}$ between the type IV and type II emission locations when the type IV attains its lowest frequency. This suggests that the energetic electrons responsible for the type IV burst might come from the continued reconnection occurring beneath the CME. The intensity of the associated soft X-ray flare has already decayed considerably at the time of the lowest frequency of the type IV burst, suggesting that the higher energy electrons contributing to plasma heating may not be accelerated in large numbers even though lower energy electrons responsible for the type IV bursts are accelerated in the reconnection region. Leblanc et al. [2000] suggested that a shock wave propagating behind the associated CME populated the CME loops with energetic electrons that emitted the type IV burst. This interpretation once again relies on blast waves not driven by the associated CME. Ground-based observatories reported type II burst activity only during a short interval (09:43 to 09:47 UT), which is unlikely to be providing energetic electrons for the extended type IV burst.

Figure 10 shows some recent statistics on the type IV bursts confirming the earlier results obtained for a smaller number of events [Gopalswamy, 2004c]. The average speed of CMEs associated with type IV bursts is clearly very high, similar to that of CMEs associated with $\mathrm{m}$ - km type II bursts and large SEP events. The fraction of halos (78\%) and the width of the non-halos $\left(166^{\circ}\right)$ indicate that very high energy CMEs are involved in these bursts. The minimum frequency attained by the type IV bursts ranges from $\sim 2 \mathrm{MHz}$ to the maximum frequency $(\sim 14 \mathrm{MHz})$ of the WAVES spectral range, with an average value of $6.6 \mathrm{MHz}$. Another remarkable result in Fig. 10 is that there is no obvious solar-cycle variation of the type IV occurrence rate. The rate steadily increases through the declining phase of solar cycle 23 and drops to zero only during the solar minimum (in the year 2007). The type IV bursts are the rarest among the three types of low-frequency radio bursts: only 42 occurred over a period of 12 years (3.5 bursts per year) since the launch of the Wind mission. Most of the type IV events are associated with SEPs, but large SEP events such as the famous Bastille Day event of 2000 (July 14) was not associated with a DH type IV burst. Thus currently it remains an open question as to what circumstances leads 
to the extension of the type IV bursts to low frequencies.

\section{Conclusions}

The low-frequency radio bursts discussed in this paper are rare, distinct, and prominent in the dynamic spectrum. All the three types of radio bursts are associated with energetic CMEs, with the average speeds generally exceeding $1000 \mathrm{~km} / \mathrm{s}$. High energy CMEs can propagate farther into the IP medium and have serious consequences throughout the heliosphere. Radio bursts can be very useful in indicating SEP events because the radio waves can travel to Earth in $\sim 8.3 \mathrm{~min}$, while $\sim 100 \mathrm{MeV}$ protons take $\sim 23 \mathrm{~min}$ to reach Earth (assuming a path length of $\sim 1.2 \mathrm{AU}$ ). There are also important differences among the radio bursts, especially in the origin of the energetic electrons responsible for the bursts, and the topology of the magnetic structures into which the electrons propagate. Unfortunately, these bursts are not spatially resolved, so some of the inferences made in the paper cannot be confirmed. Future low-frequency imaging arrays are expected to greatly advance our understanding of the bursts and their relation to CMEs.

Acknowledgments. The author thanks P. Mäkelä, S. Yashiro, and S. Akiyama for help with the manuscript preparation. Work supported by NASA Living with a Star Program.

\section{References}

Aguilar-Rodriguez, E., N. Gopalswamy, R. J. MacDowall, S. Yashiro, and M. L. Kaiser, A Study of the Drift Rate of Type II Radio Bursts at Different Wavelengths, Proceedings of Solar Wind 11/SOHO 16, 393-396, 2005.

Boischot, A., A.C. Riddle, J. B. Pearce, and J.W. Warwick, Shock waves and type II radiobursts in the interplanetary medium, Solar Phys., 65, 397-404, 1980.

Bougeret, J.-L., M. L. Kaiser, P. J. Kellogg, R. Manning, K. Goetz, S. J. Monson, N. Monge, L. Friel, C. A. Meetre, C. Perche, L. Sitruk and S. Hoang, Waves: The Radio and Plasma Wave Investigation on the Wind Spacecraft, Space Sci. Rev., 71, 231-263, 1995.

Bougeret, J.-L., P. Zarka, C. Caroubalos, M. Karlicky, Y. Leblanc, D. Maroulis, A. Hillaris, X. Moussas, C. E. Alissandrakis, G. Dumas and C. Perche, A shock associated (SA) radio event and related phenomena observed from the base of the solar corona to 1 AU, Geophys. Res. Lett., 25, 2513-2516, 1998.

Brueckner, G. E., R. A. Howard, M. J. Koomen, C. M. Korendyke, D. J. Michels, J. D. Moses, D. G. Socker, K. P. Dere, P. L. Lamy, A. Lebaria, M. V. Bout, R. Schwenn, G. M. Simnett, D. K. Bedford, and C. J. Eyles, The Large Angle Spectroscopic Coronagraph (LASCO), Solar Phys., 162, 357-402, 1995.

Cane, H.V., and R. G. Stone, Type II solar radio bursts, interplanetary shocks, and energetic particle events, Astrophys. J., 282, 339-344, 1984. 
Cane, H. V., and W. C. Erickson, Solar Type II Radio Bursts and IP Type II Events, Astrophys. J., 623, 1180-1194, 2005.

Cane, H. V., R. G. Stone, J. Fainberg, J. L. Steinberg, S. Hoang, and R. T. Stewart, Radio evidence for shock acceleration of electrons in the solar corona, Geophys. Res. Lett., 8, 1285-1288, 1981.

Cane, H. V., N.R. Sheeley, and R. A. Howard, Energetic interplanetary shocks, radio emission, and coronal mass ejections, J. Geophys. Res., 92, 9869, 1987.

Cane, H. V., W. C. Erickson, and N. Prestage, Solar flares, type III radio bursts, coronal mass ejections, and energetic particles, J. Geophys. Res., 107, SSH 14-1, 2002.

Cliver, E. W. and A. G. Ling, Low-Frequency Type III Bursts and Solar Energetic Particle Events, Astrophys. J., 690, 598-609, 2009.

Cliver, E. W., D. F. Webb, and R. A. Howard, On the origin of solar metric type II bursts, Solar Phys., 187, 89-114, 1999.

Cliver, E. W., S. W. Kahler, and D. V. Reames, Coronal shocks and solar energetic proton events, Astrophys. J., 605, 902-910, 2004.

Dulk, G. A., Y. Leblanc, T.S. Bastian, and J.-L. Bougeret, Acceleration of electrons at type II shock fronts and production of shock-accelerated type III bursts, J. Geophys. Res., 105, 27343-27352, 2000.

Erickson, W. C., The Bruny Island radio spectrometer, Pub. Astron. Soc. Australia, 14, 3, $278-282,1997$.

Ginzburg, V. L., and V. V. Zheleznyakov, On the Possible Mechanisms of Sporadic Solar Radio Emission (Radiation in an Isotropic Plasma), Soviet Astron., 2, 653, 1958.

Gopalswamy, N., Solar and geospace connections of energetic particle events, Geophys. Res. Lett., 30, 8013, 2003.

Gopalswamy, N., Interplanetary radio bursts, in Solar and Space Weather Radiophysics, edited by C. U. Keller and D. E. Gary, 305, 2004a.

Gopalswamy, N., A Global Picture of CMEs in the Inner Heliosphere, in The Sun and the Heliosphere as an Integrated System, edited by G. Poletto and S. T. Suess, 201, $2004 \mathrm{~b}$.

Gopalswamy, N., Recent advances in the long-wavelength radio physics of the Sun, Planet. Space Sci., 52, 1399-1413, 2004c.

Gopalswamy, N., Coronal Mass Ejections and Type II Radio Bursts, Geophys. Monogr. Ser., 165, 207, 2006.

Gopalswamy, N., and P. Mäkelä, Long-duration low-frequency type III bursts and solar energetic particle events, Astrophys. J. Lett., 721, L62-L66, 2010.

Gopalswamy, N., M.L. Kaiser, B. J. Thompson, L. F. Burlaga, A. Szabo, A. Vourlidas, A. Lara, S. Yashiro and J.-L. Bougeret, Radio-rich Solar Eruptive Events, Geophys. Res. Lett., 27, 1427, 2000. 
Gopalswamy, N., A. Lara, M. L. Kaiser, and J.-L. Bougeret, Near-Sun and near-Earth manifestations of solar eruptions, J. Geophys. Res., 106, 25261-25278, 2001a.

Gopalswamy, N., S. Yashiro, M. L. Kaiser, R. A. Howard, and J.-L. Bougeret, Characteristics of coronal mass ejections associated with long-wavelength type II radio bursts, J. Geophys. Res., 106, 29219-29230, 2001b.

Gopalswamy, N., E. Aguilar-Rodriguez, S. Yashiro, S. Nunes, M. L. Kaiser, and R. A. Howard, Type II radio bursts and energetic solar eruptions, J. Geophys. Res., 110, A12S07, 2005.

Gopalswamy, N., S. Yashiro, H. Xie, S. Akiyama, E. Aguilar-Rodriguez, M. L. Kaiser, R. A. Howard, and J.-L. Bougeret, Radio-Quiet Fast and Wide Coronal Mass Ejections, Astrophys. J., 674, 560, 2008a.

Gopalswamy, N., S. Yashiro, S. Akiyama, P. Mäkelä, H. Xie, M. L. Kaiser, R. A. Howard, and J.-L. Bougeret, Coronal mass ejections, type II radio bursts, and solar energetic particle events in the SOHO era, Ann. Geophys., 26, 3033-3047, 2008b.

Gopalswamy, N., S. Akiyama, and S. Yashiro, Major solar flares without coronal mass ejections, in Universal Heliophysical Processes, edited by N. Gopalswamy and D. F. Webb, 283-286, 2009a.

Gopalswamy, N., W. T. Thompson, J. M. Davila, M. L. Kaiser, S. Yashiro, P. Mäkelä, G. Michalek, J.-L. Bougeret, and R. A. Howard, Relation between type II bursts and CMEs inferred from STEREO observations, Solar Phys., 259, 227-254, 2009b.

Gopalswamy, N., H. Xie, P. Mäkelä, S. Akiyama, S. Yashiro, M. L. Kaiser, R. A. Howard, and J.-L. Bougeret, Interplanetary shocks lacking type II radio bursts, Astrophys. J., 710, 1111-1126, 2010b.

Kahler, S. W., E. W. Cliver, and H. V. Cane, The relationship of shock-associated hectometric emission with metric type II bursts and energetic particles, Adv. Space Res., 6, 319-322, 1986.

Klein, K.-L., G. Trottet, and A. Klassen, Energetic Particle Acceleration and Propagation in Strong CME-Less Flares, Solar Phys., 263, 185-208, 2010.

Kundu, M. R., and R. G. Stone, Observations of solar radio bursts from meter to kilometer wavelengths, Adv. Space Res., 4, 261-270, 1984.

Leblanc, Y., G. A. Dulk, and J.-L. Bougeret, Tracing the Electron Density from the Corona to 1 AU, Solar Phys., 183, 165-180, 1998.

Leblanc, Y., G. A. Dulk, I. H. Cairns, and J.-L. Bougeret, Type II flare continuum in the corona and solar wind, J. Geophys. Res., 105, 18215-18223, 2000.

Lengyel-Frey, D. and R. G. Stone, Characteristics of interplanetary type II radio emission and the relationship to shock and plasma properties, J. Geophys. Res., 94, 159-167, 1989 .

MacDowall, R. J., A. Lara, P. K. Manoharan, N. V. Nitta, A. M. Rosas, and J.-L. Bougeret, Long-duration hectometric type III radio bursts and their association with solar energetic particle (SEP) events, Geophys. Res. Lett., 30, SEP 6-1, 2003. 
Magdalenić, J., C. Marqué, A. N. Zhukov, B. Vršnak, and T. Žic, Origin of Coronal Shock Waves Associated with Slow Coronal Mass Ejections, Astrophys. J., 718, 266-278, 2010 .

Malitson, H. H., J. Fainberg, and R. G. Stone, Observation of a Type II Solar Radio Burst to 37 Rsun, Astrophys. Lett., 14, 111-114, 1973.

Mann, G., and A. Klassen, Electron beams generated by shock waves in the solar corona, Astron. Astrophys., 441, 319-326, 2005.

Mann, G., A. Klassen, H. Aurass, and H.-T. Classen, Formation and development of shock waves in the solar corona and the near-Sun interplanetary space, Astron. Astrophys., 400, 329-336, 2003.

Melnik, V. N., H. O. Rucker, A. A. Konovalenko, V. V. Dorovskyy, E. P. Abranin, A. I. Brazhenko, B. Thide, A. A. Stanislavskyy, Solar Type IV Bursts at Frequencies 10-30 $\mathrm{MHz}$, in Solar Physics Research Trends, edited by P. Wang, Nova Science Publishers, New York, 2008.

Munro, R. H., J. T. Gosling, E. Hildner, R. M. MacQueen, A. I. Poland, and C. L. Ross, The association of coronal mass ejection transients with other forms of solar activity, Solar Phys., 61, 201-215, 1979.

Newkirk, G., Structure of the solar corona, Ann. Rev. Astron. Astrophys., 5, 213, 1967.

Payne-Scott, R., D. E. Yabsley, and J. G. Bolton, Relative Times of Arrival of Bursts of Solar Noise on Different Radio Frequencies, Nature, 160, 256-257, 1947.

Raymond, J. C., B. J. Thompson, O. C. St. Cyr, N. Gopalswamy, S. Kahler, M. Kaiser, A. Lara, A. Ciaravella, M. Romoli, and R. O'Neal, SOHO and radio observations of a CME shock wave, Geophys. Res. Lett., 27, 1439-1442, 2000.

Reiner, M. J., M. Karlický, K. Jiřička, H. Aurass, G. Mann, and M. L. Kaiser, On the Solar Origin of Complex Type III-like Radio Bursts Observed at and below 1 MHZ, Astrophys. J., 530, 1049-1060, 2000.

Robinson, R. D., The relation between flare-related metric continuum bursts and coronal mass ejections, Solar Phys., 104, 33-39, 1986.

Saito, K., R. Munro, and A. Poland, A study of the background corona near solar minimum, Solar Phys., 55, 121-134, 1977.

Sheeley, Jr. N. R., R. A. Howard, D. J. Michels, R. D. Robinson, M. J. Koomen, and R. T. Stewart, Associations between coronal mass ejections and metric type II bursts, Astrophys. J., 279, 839-847, 1984.

Uchida, Y., On the Exciters of Type II and Type III Solar Radio Bursts, Pub. Astron. Soc. Japan, 12, 376-397, 1960.

Vršnak, B., H. Aurass, J. Magdalenic, and N. Gopalswamy, Band-splitting of coronal and interplanetary type II bursts. I. Basic properties, Astron. Astrophys., 377, 321-329, 2001. 
Wild, J.P., Observations of the Spectrum of High-Intensity Solar Radiation at Metre Wavelengths. III. Isolated Bursts, Aust. J. Sci. Res. A, 3, 541-557, 1950.

Wild, J.P. and L. L. McCready, Observations of the Spectrum of High-Intensity Solar Radiation at Metre Wavelengths. I. The Apparatus and Spectral Types of Solar Burst Observed, Aust. J. Sci. Res. A, 3, 387-398, 1950. 